\title{
Mitral Multifocal Papillary Fibroelastoma in a Patient with Aggressive Fibromatosis
}

\author{
Philipp P. Müller ${ }^{1}$ Abbas Agaimy ${ }^{2}$ Michael Weyand ${ }^{1}$ Christian Heim ${ }^{1}{ }^{(0)}$
}

${ }^{1}$ Department of Cardiac Surgery, Friedrich-Alexander University Erlangen-Nuremberg, Erlangen, Germany

2 Institute of Pathology, Friedrich-Alexander University ErlangenNuremberg, Erlangen, Germany

\author{
Address for correspondence Christian Heim, MD, MHBA, Department \\ of Cardiac Surgery, Friedrich-Alexander University Erlangen- \\ Nuremberg, Krankenhausstr. 12, 91054 Erlangen, Germany \\ (e-mail: Christian.Heim@FAU.de).
}

Thorac Cardiovasc Surg Rep 2021;10:e61-e63.

\begin{abstract}
Keyword

- histology

- mitral valve surgery

- tumor

- cardiac

Mitral valve fibroelastoma is a rare condition that can be associated with high morbidity rates due to thrombus formation and resulting embolic events. Causative treatment for affected patients is mitral valve surgery. An association between cardiac fibroelastoma and desmoid-type fibromatosis, an aggressive form of fibromatosis, is not yet described. We present a case of a 58-year-old man with a history of desmoid-type fibromatosis and concomitant papillary fibroelastoma of the mitral valve who consequently underwent mitral valve replacement.
\end{abstract}

\section{Introduction}

Cardiac tumors are rare with an overall incidence rate of $<0.5 \%$. They are classified by their origin (primary tumor vs. metastasis) and their dignity (benign vs. malignant). Eight percent of primary cardiac tumors are benign and of these, fibroelastomas (papillary fibroelastoma [PFE]) account for $16 \%$ of all lesions. ${ }^{1}$

PFEs originate from the endocardium and are most commonly found on the heart valve leaflets. Clinical implications of these lesions can be various and range from asymptomatic to massive complications such as embolic stroke. Echocardiographic assessment is the most important diagnostic tool for intracardiac masses. A multidisciplinary approach including surgeons, cardiologists, pathologists, and radiologists in diagnosis and treatment is important in finding an optimal therapeutic strategy for each individual patient. ${ }^{2}$ Distinguishing PFE from giant Lambl's excrescences might be arbitrary or even impossible and a disease continuum has been suggested.

Desmoid tumors (aggressive fibromatosis) are tumors deriving from mesenchymal stem cells, characterized by a locally infiltrating, nonmetastasizing behavior. There is evidence that tumor formation is linked to mutations either in CTNNB1/ $\beta$-catenin (in sporadic cases) or in the adenomatous polyposis coli (APC) gene (mostly in hereditary cases) leading to dysregulated Wingless/Int-1/APC/ $\beta$-catenin pathway. Mutations in the APC gene are responsible for the development of familial adenomatous polyposis (FAP), an autosomal dominant inherited condition which leads to the development of a multitude of polyps, especially in the large intestine. ${ }^{3}$ Gardner's syndrome, a subtype of FAP, is characterized by the presence of multiple polyps in the colon together with tumors outside the colon and carries a high risk for the development of colorectal cancer.

Diagnosis of desmoid tumors is made by magnetic resonance imaging (MRI) and histopathological studies, while treatment options are determined by location and size of the tumor and other individual variables and should be planned cautiously in a multidisciplinary team approach. There is no previously reported association between PFEs of the mitral valve and aggressive fibromatosis. received

March 30, 2021

accepted after revision

June 29, 2021
DOI https://doi.org/

10.1055/s-0041-1736208. ISSN 2194-7635. (c) 2021. The Author(s).

This is an open access article published by Thieme under the terms of the Creative Commons Attribution-NonDerivative-NonCommercial-License, permitting copying and reproduction so long as the original work is given appropriate credit. Contents may not be used for commercial purposes, or adapted, remixed, transformed or built upon. (https://creativecommons.org/ licenses/by-nc-nd/4.0/) Georg Thieme Verlag KG, Rüdigerstraße 14, 70469 Stuttgart, Germany 


\section{Case Presentation}

A 58-year-old man was admitted to our cardiac surgery department from an external hospital with suspected endocarditis of the mitral valve. The patient was initially hospitalized due to impaired vision which he reported to emerge abruptly the night before. There were no signs of fever or recent weight loss reported by the patient at admission.

Medical history of the patient included atrial flutter, aneurysm of the left atrium, multiple sclerosis treated with immunosuppressive therapy, indolent non-Hodgkin B cell lymphoma, desmoid fibromatosis of the thorax resected 2 years earlier, and multiple gallbladder polyps. Additionally, the patient had a family history suspicious for FAP. His father died of colorectal cancer, but the patient himself denied genetic testing.

After admission, a cerebral MRI was performed which showed disseminated embolic strokes in the territory of the posterior cerebral artery. To rule out endocarditis, echocardiography was performed, showing floating structures on the leaflets of the mitral valve (-Fig. 1). Blood tests showed slightly elevated leukocytes with all other inflammatory values within normal limits. Additionally, blood cultures were taken and intravenous antibiotic therapy was started. Despite negative results from the blood cultures, the patient was then admitted to our clinic for minimally invasive mitral valve replacement (three-dimensional endoscopic via right lateral thoracotomy) due to morphologically unchanged structures on the mitral leaflets and the high embolic risk (-Fig. 2). Intraoperatively, two cauliflower-like lesions on the anterior and posterior mitral valve leaflets were resected (-Fig.3). The specimens showed a sea anemone-like appearance when placed in saline and were taken for histological and microbiological studies. The resection of both lesions adherent to the free edge of the anterior and posterior leaflets included the removal of primary chordae and the extend of defects hampered valve reconstruction. Therefore, mitral valve replacement was performed preserving the entire subvalvular apparatus to maintain annular-papillary continuity. Due to the patient's explicit request, a biological prosthesis (Edwards Perimount Magna Mitral Ease $31 \mathrm{~mm}$ ) was used for replacement as the patient was rejecting

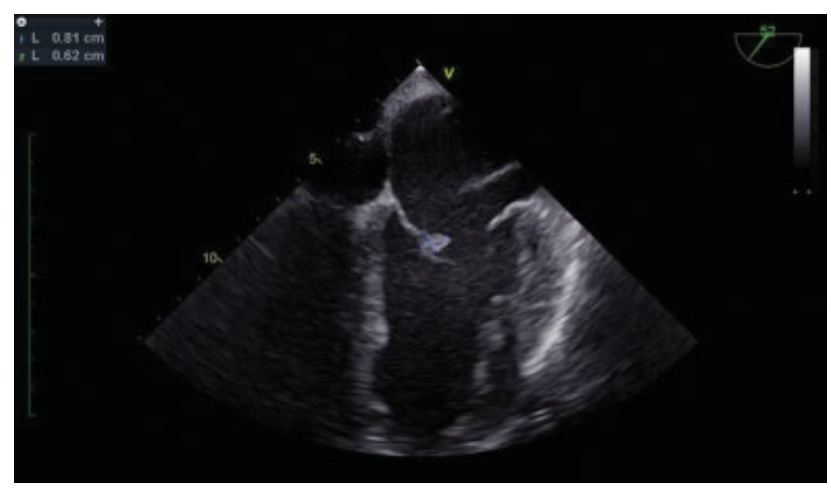

Fig. 1 Transesophageal echocardiography (mid-esophageal four-chamber view) of the mitral valve showing a floating structure on the anterior leaflet.

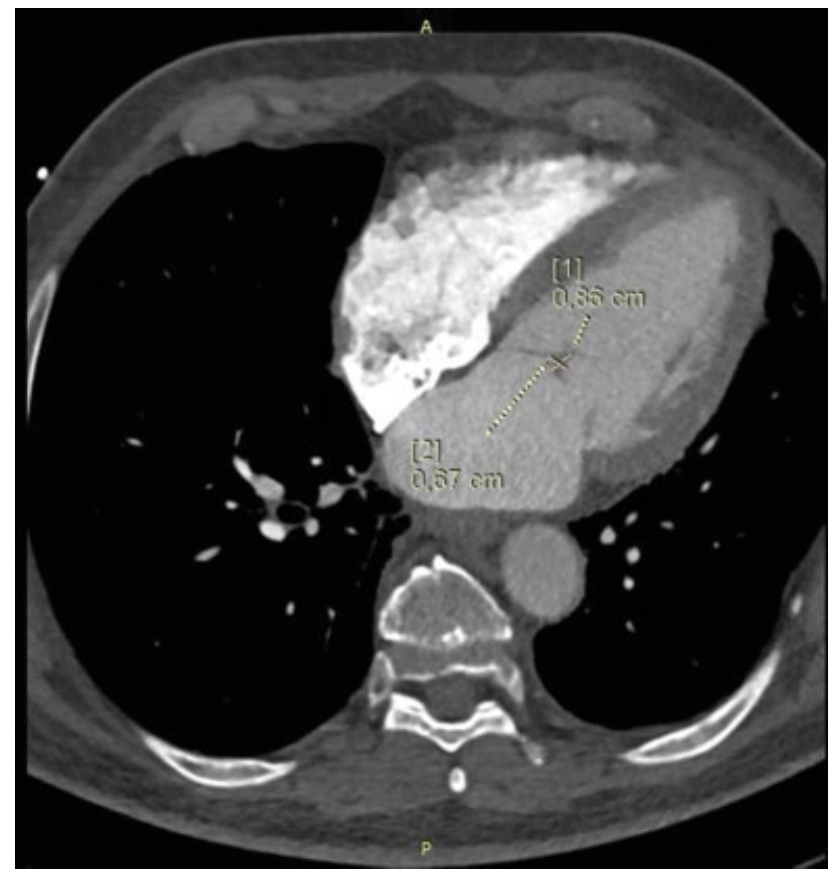

Fig. 2 Computed tomography of the thorax (Siemens Somatom Definition Edge) with contrast agent (Solutrast 370, lopamidol) showing a solid structure on the mitral valve.

postoperative intake of oral anticoagulation. The postoperative course was uneventful and he showed no signs of irreversible neurological symptoms. Blood cultures and microbiological studies were negative for bacterial pathogens. Histopathological investigations showed irregular branching papillae composed of fibroelastic tissue covered by normal endocardium, consistent with PFEs with predominance of fibrous stroma (-Fig. 4). Immunohistochemistry revealed no aberrant nuclear $\beta$-catenin expression in the stromal cells. Therefore, intravenous antibiotic therapy for endocarditis was stopped and the patient was informed about the condition. The patient denied further genetic testing or coloscopy at this time and was discharged home in good clinical condition 10 days after the surgery ( - Fig. 4 ).

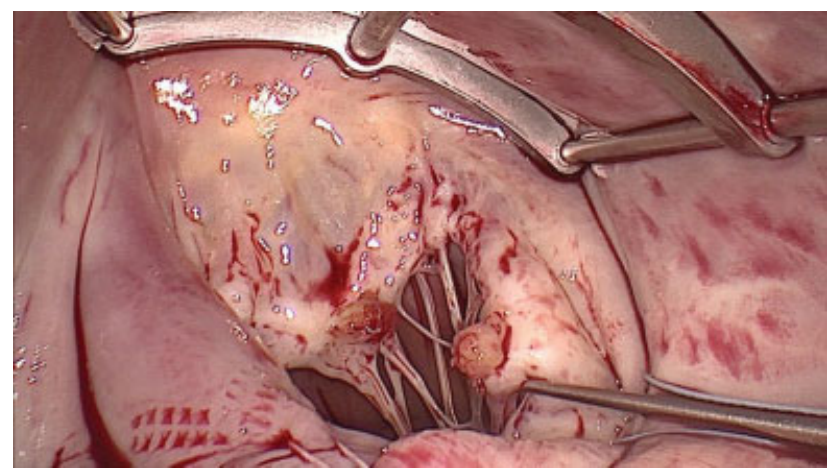

Fig. 3 Operative situs during minimally invasive cardiac mitral valve surgery. Cauliflower-like adhesive lesions on the A2 segment of the anterior mitral valve leaflet and on the $\mathrm{P} 3$ segment of the posterior leaflet were resected. The mitral valve was replaced with a biological prothesis (Edwards Perimount Magna Mitral Ease $31 \mathrm{~mm}$ ) preserving the entire subvalvular apparatus. 


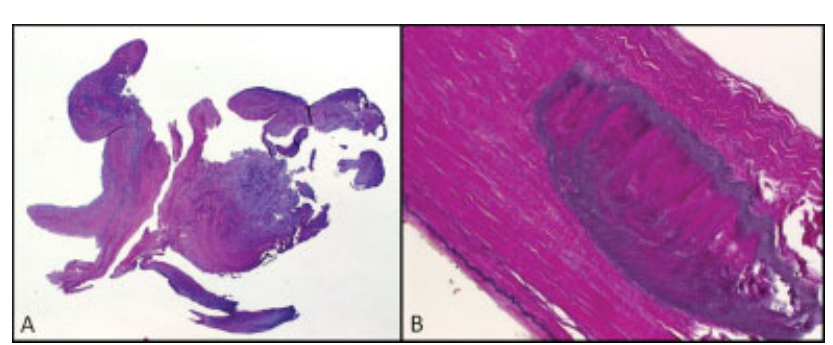

Fig. 4 (A) Histopathological analysis (hematoxylin and eosin stain) of the specimen showing irregularly branching papillae (magnification $\times 50$ ) composed of fibroelastic tissue. (B) $\times 200$ magnification (elastic Van Gieson stain) showing characteristic triphasic pattern with a prominent elastic central core within the papillae.

\section{Discussion}

There is no reported association between PFEs of the mitral valve and aggressive fibromatosis. Nevertheless, we suspect a relationship of these conditions in this case as the patient was suffering from a desmoid tumor, a condition associated with a mutation of the APC gene., ${ }^{3,4}$ The same gene is responsible for the development of FAP and Gardner's syndrome, which is a phenotypical variant of FAP. Despite the fact that the patient never underwent genetic testing, it is likely that he carries the mutation considering his family history, the autosomal dominate course of its inheritance and his other conditions. Gardner's syndrome is associated with the development of benign tumors that can emerge on multiple locations. Cardiac involvement by FAP/Gardner's syndrome has not been described; nevertheless, a multifocal tumor development in desmoid-type fibromatosis was previously described. ${ }^{4}$ Patients with these conditions require extensive tests to rule out involvement of organ systems, including the heart. Interestingly, a desmoid-type tumor in a patient with Gardner's syndrome was associated with left atrial aneurysm in another case report, ${ }^{5}$ the same condition our patient was suffering from. This would be the second case an association between these conditions was described in literature. Whether the association between cardiac disease and desmoid tumors (or FAP) is coincidental or etiological remains speculative.

Despite the benign nature of primary cardiac tumors in most cases, they are associated with high rates of morbidity due to infiltrating growth and embolic events such as stroke. Therefore, surveillance, and if necessary surgical removal, is crucial in preventing catastrophic outcomes in affected patients. This case draws additional attention to a possible cardiac involvement in patients with a mutation in the APC gene.

\section{Conclusion}

Primary cardiac tumors are a rare condition but associated with a high symptom burden for patients. A correlation between mutations in APC and its associated syndromes is not yet described. In this case, we assume a coherency between the patient's predisposition for developing tumors on multiple locations and a cardiac involvement. Patients with FAP/Gardner's syndrome necessitate extensive surveillance and a multidisciplinary approach to reduce complications.

\section{Authors' Contribution}

Concept/design: C.H.; data interpretation: A.A. and P.P.M.; drafting article: P.P.M.; critical revision of article: C.H.; approval of article: P.P.M., A.A., M.W., and C.H.; funding: C.H.

\section{Institutional Review Board Waiver and Patient Consent} Statement

Patient signed informed consent related to clinical course; therefore and due to its retrospective nature of the educational case report, the IRB was waived.

\section{Funding}

This publication was supported by Deutsche Forschungsgemeinschaft and University of Erlangen Foundation within the funding program Open Access Publishing.

Conflict of Interest

None declared.

\section{References}

1 Ren DY, Fuller ND, Gilbert SAB, Zhang Y. Cardiac tumors: clinical perspective and therapeutic considerations. Curr Drug Targets 2017;18(15):1805-1809

2 Poterucha TJ, Kochav J, O'Connor DS, Rosner GF. Cardiac tumors: clinical presentation, diagnosis, and management. Curr Treat Options Oncol 2019;20(08):66

3 Penel N, Chibon F, Salas S. Adult desmoid tumors: biology, management and ongoing trials. Curr Opin Oncol 2017;29(04): 268-274

4 Skubitz KM. Biology and treatment of aggressive fibromatosis or desmoid tumor. Mayo Clin Proc 2017;92(06):947-964

5 Mole MT, Goldstraw P, Sheppard MN. Desmoid tumour in thoracotomy scar 5 years after excision of a left giant atrial appendage aneurysm in female with a family history of Gardner's syndrome. Thorac Cardiovasc Surg 1992;40(05): 300-302 